\title{
THE DISTURBANCE OF OXIDATIVE PHOSPHORYLATION BY N-ACETOXY-N-ACETYL-2-AMINOFLUORENE, A MODEL ULTIMATE CARCINOGEN*
}

\author{
Herbert I. Hadler and John M. Demetriou \\ Department of Chemistry and Biochemistry, Southern Illinois University \\ Carbondale, Illinois 62901, U.S.A.
}

(Received for publication June 23, 1975)

\begin{abstract}
Currently $\mathrm{N}$-acetoxy-N-acetyl-2-aminofluorene is favored by many investigators to be a model of the ultimate electrophilic carcinogenic agent derived metabolically from the carcinogen $\mathrm{N}$-acetyl-2-aminofluorene. The model induced in vitro a delayed ATP energized increase in mitochondrial volume as indicated by the decrease in absorbancy at $520 \mathrm{~nm}$. The ATP energized decrease in absorbancy was inhibited by rutamycin, 2,4dinitrophenol and a high level of antimycin known to induce ATPase activity. The ATP energized effect was not altered either by rotenone or by a low level of antimycin known to inhibit respiration without inducing ATPase activity. Malate or potassium ion did not affect the phenomenon, however, sulfate ion which has been implicated in liver carcinogenesis shortened the induction period. Showdomycin stimulated the phenomenon. N-Acetoxy-N-acetyl-2-aminofluorene interacts with the machinery of oxidative phosphorylation. $\mathrm{N}$-Acetoxy-N-acetyl-2-aminofluorene was enzymically converted by the mitochondria to $\mathrm{N}$-hydroxy-N-acetyl-2-aminofluorene. These findings extend the experimental confluence of oxidative phosphorylation with carcinogenesis.
\end{abstract}

A comprehensive examination of the action of carcinogens, metabolites of carcinogens and related derivatives of carcinogens with tissue constiuents should lead to meaningful data regarding the mechanism of chemical carcinogenesis. As a result of this general approach we synthesized a variety of K-region derivatives of the potent carcinogen 7,12-dimethylbenz (a) anthracene over a decade ago ${ }^{11}$. We have also examined the interaction of several carcinogens and their metabolites with mitochondria by means of a convenient in vitro system based upon the ability of an agent or a combination of agents to induce an ATP-energized mitochondrial volume change. The change was indicated by a decrease in absorbancy at $520 \mathrm{~nm}$. While the three parent carcinogens, N-acetyl-2-aminofluorene (AAF), dibenz $(\mathrm{a}, \mathrm{h})$ anthrancene and $\mathrm{N}$-acetyl-4aminobiphenyl were inactive in this in vitro system; their appropriate acidic metabolites, $\mathrm{N}$ hydroxy-N-acetyl-2-aminofluorene (N-OH-AAF), 4', , ' -dihydroxy-1,2,5,6-dibenz-9,10-anthraquinone and $\mathrm{N}$-hydroxy-N-acetyl-4-aminobiphenyl were active in this in vitro test ${ }^{2,3,4}$. The studies with $\mathrm{N}-\mathrm{OH}-\mathrm{AAF}$ were extended to three other isomers in which constituents were not only in position two of the fluorenyl moiety as in N-OH-AAF but also in positions one, three and four ${ }^{5}$. The four N-hydroxy derivatives induced (when combined with showdomycin) ATP-energized mitochondrial changes whose magnitudes were parallel to their carcinogenicity in the rat. These in vitro mitochondrial studies illustrated a confluence of experimental data dealing with oxidative phosphorylation and chemical carcinogenesis and directed attention to our unitary hypothesis

* Supported in part by USPHS grant CA-10759 (National Cancer Institute) and the Eli Lilly Research Foundation.

We wish to thank Mrs. BARBra G. DANIEL who carried out the first experiments with N-OAc-AAF. 
of carcinogenesis ${ }^{2,6)}$. This hypothesis postulated that when a carcinogen interferes with a flux of energy in the mitochondria, mitochondrial membrane damage becomes feasible and such damage could result in the release of mitochondrial genetic material. This released mitochondrial genetic material could then behave like an oncogenic virus and enter the genome of the cell.

On the other hand Miller and Miller have suggested for some time ${ }^{7,81}$ that the critical reaction which engenders carcinogenesis was between a nucleophilic site of the informational macromolecules (proteins and nucleic acids) and an electrophilic site of the original carcinogen or appropriate metabolite derived from the carcinogen. The crucial electrophilic species was named the ultimate carcinogen while intermediates between the parent carcinogen and the ultimate carcinogen were designated as proximate carcinogens. Miller and MILLer and associates $^{9,10,11,7,8)}$ and others ${ }^{12,13)}$ designated in rat liver, N-OH-AAF to be the proximate carcinogen and $\mathrm{N}-\mathrm{OSO}_{2} \mathrm{OH}-\mathrm{AAF}$ to be the ultimate carcinogen derived from AAF. Because of the considerable lability of $\mathrm{N}-\mathrm{OSO}_{2} \mathrm{OH}-\mathrm{AAF}$ much experimental work had been carried out with the more stable and more lipid-soluble anhydride $\mathrm{N}$-acetoxy-N-acetyl-2-aminofiuorene ( $\mathrm{N}$ OAc-AAF $)^{14,9,15,10,16,7,17,18,19,20)}$. Other workers have also considered the possibility that N-OAcAAF may be a metabolite of N-OH-AAF because (a) the slow nonenzymic acetylation of $\mathrm{N}$ $\mathrm{OH}-\mathrm{AAF}$ by acetyl-CoA ${ }^{21 \prime}$ and (b) the enzymic conversion of N-OH-AAF to N-OAc-AAF and 2-nitorsofluorene by certain mammalian peroxidases ${ }^{22)}$. MILler and Miller also reported that $\mathrm{N}-\mathrm{OAc}-\mathrm{AAF}$ was a more potent carcinogen than N-OH-AAF${ }^{231}$.

In order to further explore the confluence of experimental observations dealing with oxidative phosphorylation and carcinogenesis we have examined the in vitro interaction of N-OAc-AAF with mitochondria by means of our ATP energized mitochondrial volume change system and also by means of oxygen uptake and ATPase measurements.

\section{Materials and Methods}

General procedures, methods and purification of the water and preparation of mitochondria has been described ${ }^{24,25,26,3)}$ for the mitochondrial volume change experiments.

Mitochondrial Swelling

Incubation was at $27^{\circ} \mathrm{C}$ in standard rectangular glass cuvettes with a $1-\mathrm{cm}$ light path. The basic reaction mixture for the volume change experiments had a final volume of $3 \mathrm{ml}$ and contained $0.75 \mathrm{mg}$ mitochondrial protein (prepared fresh daily from male rat liver), $75 \mathrm{~mm}$ sucrose and $75 \mathrm{~mm}$ tris- $\mathrm{HCl}$ buffer of $\mathrm{pH} 7.4$. A decrease in absorbancy at $520 \mathrm{~nm}$ was taken as a measure of mitochondrial swelling ${ }^{24)}$. A model 2000 automatic spectrophotometer manufactured by Gilford Instrument Laboratories, Inc., Oberlin, Ohio, was used. All cations were added in the form of chloride salts and anions were added in a form of tris salts neutralized to $\mathrm{pH} 7.4$. $\mathrm{N}-\mathrm{OH}-\mathrm{AAF}$ and N-OAc-AAF were clissolved in acetone (dried with sodium sulfate and distilled from potassium permanganate) or dimethylsulfoxide (distilled from calcium hydride in vacuo and stored under nitrogen) when added to the incubation mixture. These solutions were prepared fresh for each day of experimentation. All controls contained the appropriate amount of carrier solvent. The adding-mixing device has been described ${ }^{25)}$. The charts and legends. provide additional experimental details.

Oxygen Uptake

Oxygen consumption was measured at $27^{\circ} \mathrm{C}$ in an oxygen monitor which utilized a CLARK electrode. The instrument (Model 535A) was manufactured by Yellow Springs Instrument Company, Yellow Springs, Ohio. A ten inch recorder (Model 100S) manufactured by Beckman. Instruments, Fullerton, California, was used in conjunction with the oxygen monitor. 
ATPase Measurement

In the ATPase experiment, orthophosphate was measured as previously described ${ }^{24,31}$. Aliquots of $1 \mathrm{ml}$, were removed at 3 -minute intervals from the specially designed optical cuvettes by means of a spring loaded Cornwall glass syringe fitted with a $6^{\prime \prime}, 20$ gauge stainless. steel needle.

Metabolism of N-OAc-AAF

To study the enzymic metabolism of N-OAc-AAF by rat liver mitochondria, $3 \mathrm{mg}$ N-OAcAAF $(10.7 \mu$ mole $)$ in acetone $(0.1 \mathrm{ml})$ was added to the basic reaction mixture in a $16 \times 150 \mathrm{ml}$ test tube which contained $9 \mathrm{mg}$ mitochondrial protein, $75 \mathrm{~mm}$ sucrose and $75 \mathrm{~mm}$ tris- $\mathrm{HCl}$ buffer in a final volume of a $3 \mathrm{ml}$. The reaction temperature was $27^{\circ} \mathrm{C}$; shaking was at the rate of 27 excursions per minute. Following the addition of N-OAc-AAF aliquots of $1 \mathrm{ml}$ were removed at 0.5 and 30 minutes with a spring loaded Cornwall glass syringe fitted with a $6^{\prime \prime}, 20$ gauge stainless steel needle. The contents of the syringe were expelled into a $16 \times 150 \mathrm{~mm}$ test tube which contained $1 \mathrm{ml}$ of $1 \mathrm{M}$ phosphoric acid to which was then added $7 \mathrm{ml}$ of a mixture of $t$ butanol - water $(75: 25)$. The contents of the tube were mixed. A volume of $8 \mathrm{ml}$ of 1,2 dichloroethane - dichloromethane $(50: 50)$ was added and the contents were mixed. Low speed centrifugation separated the two layers. An aliquot of $11 \mathrm{ml}$ (syringe) was removed from the lower (organic layer) and dried with $2 \mathrm{~g}$ of anhydrous sodium sulfate. An aliquot $(10 \mathrm{ml})$ of the organic phase was transferred to a $50-\mathrm{ml}$ round bottom flask and taken to dryness in vacuo at $40^{\circ} \mathrm{C}$ (rotary evaporator). Acetone $(0.3 \mathrm{ml})$ was added, the residue dissolved and an aliquot of $10 \mu \mathrm{l}$ was spotted on silica gel TLC plates (QF1) $250 \mu$ thick, with phosphor purchased from Quantum Industries, Fairfield, New Jersey. The plates were developed by a mixture of chloroform - methanol $(97: 3)$. The various spots were located by means of fluorescent quenching.

Synthesis of N-OAc-AAF

The procedure was a modification of that reported by GUTMANN and ERICKSON ${ }^{27)}$. A solution of $250 \mathrm{mg}$ (1.04 nmole) of N-OH-AAF in a mixture of $17 \mathrm{ml}$ of freshly distilled pyridine and $17 \mathrm{ml}$ of acetic anhydride (distilled) stood at room temperature for 24 hours under an atmosphere of nitrogen. The volatile reagents were then removed in vacuo on a rotary evaporator at $40^{\circ} \mathrm{C}$. The residue was co-evaporated six times with benzene (purified by having been shaken with sulfuric acid, washed with water, dried with sodium sulfate and distilled). The off-white solid was washed with ether (analytical grade) and cyclohexane (purified by having been shaken with sulfuric acid, washed with water, dried with sodium sulfate and distilled) and then recrystallized from cyclohexane-acetone (dried with sodium sulfate and distilled from potassium permanganate). The crystals had a melting point of $110 \sim 111^{\circ} \mathrm{C}$ (corrected microscope hot stage). Calculated for $\mathrm{C}_{17} \mathrm{H}_{15} \mathrm{NO}_{3}: \mathrm{C}, 72.58 ; \mathrm{H}, 5.37$ Found: $\mathrm{C}, 72.61 ; \mathrm{H}, 5.40$ [Analysis carried out by Galbraith Laboratories, Knoxville, Tennessee, 37291, U.S.A.]. Gutmann and ERICKson ${ }^{271}$ reported a melting point of $112 \sim 114^{\circ} \mathrm{C}$; Lotlikar, SCribner, Miller and Miller ${ }^{14)}$ reported a melting point of $112 \sim 114^{\circ} \mathrm{C}$.

Other samples of N-OAc-AAF were kindly provided by Dr. Helmut Gutmann of Minneapolis, Minnesota and by the Drug Development Branch, Division of Drug Treatment, National Cancer Institute, Washington, D.C., U.S.A. This agency also kindly provided us with samples of $\mathrm{N}-\mathrm{OH}-\mathrm{AAF}$.

The experiments reported were carried out with the sample provided by the National Cancer Institute and this sample showed only a single spot when examined by T.L.C. as described in Fig. 11 .

\section{Results}

It is seen in Fig. 1 that N-OAc-AAF at a level of $300 \mu \mathrm{M}$ when combined with $333 \mu \mathrm{M}$ ATP did elicit a delayed but significant increase in mitochondrial volume. Two possible cleavage products of N-OAC-AAF viz. N-OH-AAF and acetate each at the level of $300 \mu \mathrm{M}$ either alone or when combined with $333 \mu \mathrm{M}$ ATP did not elicit any volume change. Similar data. 
was obtained with two different samples of N-OAc-AAF kindly provided to us by Dr. Helmut Gutmann and the National Cancer Institute. The effect was identical when dimethylsulfoxide rather than acetone was used as the carrier solvent (data not shown). The onset of the swelling phenomenon varied two to three minutes from day to day (i.e. with different mitochondrial preparations) but was constant throughout any one day with the same mitochondrial preparation in any single set of parallel controlled experiments.

The ATP energized effect with N-OAc-AAF was barely detectable at a level of $200 \mu \mathrm{M}$ $\mathrm{N}-\mathrm{OAc}-\mathrm{AAF}$ but was readily induced by $300 \mu \mathrm{M}$ N-OAc-AAF (data not shown).

It is seen in Fig. 2 that the onset of the ATP energized swelling induced by N-OAc-AAF was hastened by sulfate ion while potassium ion and malate ion had no influence.

In Fig. 3 it is seen that the ATP energized effect was not inhibited by the respiratory inhibitors rotenone and antimycin at respective levels of $3 \mu \mathrm{M}$ and $0.1 \mu \mathrm{g}$ per $3 \mathrm{ml}$. These are concentrations known to inhibit mitochondrial respiration ${ }^{28)}$ with minimal ATPase activity. When the concentration of antimycin was increased tenfold to $1 \mu \mathrm{g}$ per $3 \mathrm{ml}$ the volume change effect was blocked. It is known that antimycin at high concentrations induces ATPase activity ${ }^{28}$.

Fig. 1. N-OAc-AAF, N-OH-AAF and acetate alone and combined with ATP.

The basic medium (see "Materials and Methods"') was used. Either N-OAc-AAF (in acetone) or N-OH-AAF (in acetone) or an aqueous solution of tris acetate was added in a volume of $0.03 \mathrm{ml}$ by means of the adding-mixing device as indicated by arrows. The adding-mixing device also carried $0.03 \mathrm{ml}$ of acetone when tris acetate was added. The final volume of system was $3 \mathrm{ml}$. The ATP (tris salt), where indicated, was present in the cuvette before the mitochondria were added. The top trace is a mitochondrial control with the addition of solvent. The designations in the lower right-hand corner of this and subsequent figures are for internal reference at our laboratory.

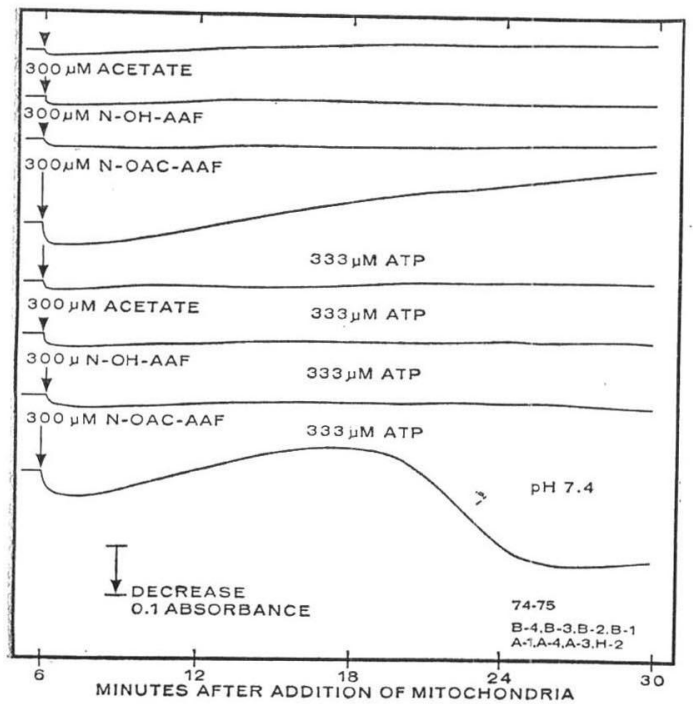

Fig. 2. The effect of various ions.

See legend of Fig. 1 for conditions. The various ions, where indicated, were present in the cuvette before the mitochondria were added.

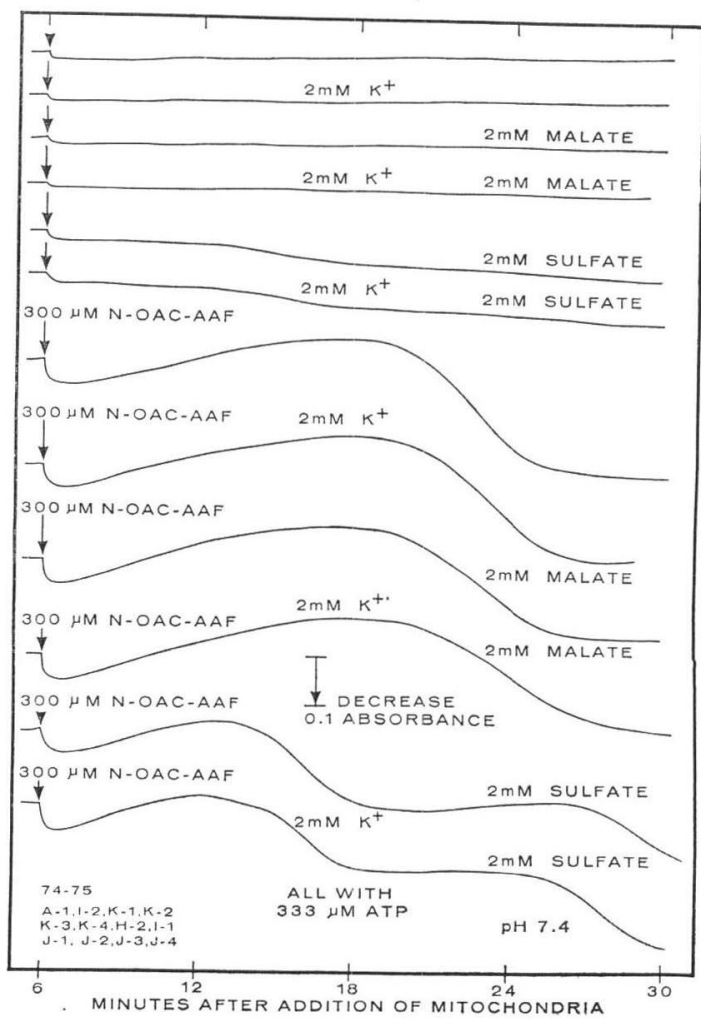


Fig. 3. The effect of various inhibitors.

See legend of Fig. 1 for conditions. The various inhibitors, where indicated, were present in the cuvette before the mitochondria were added.

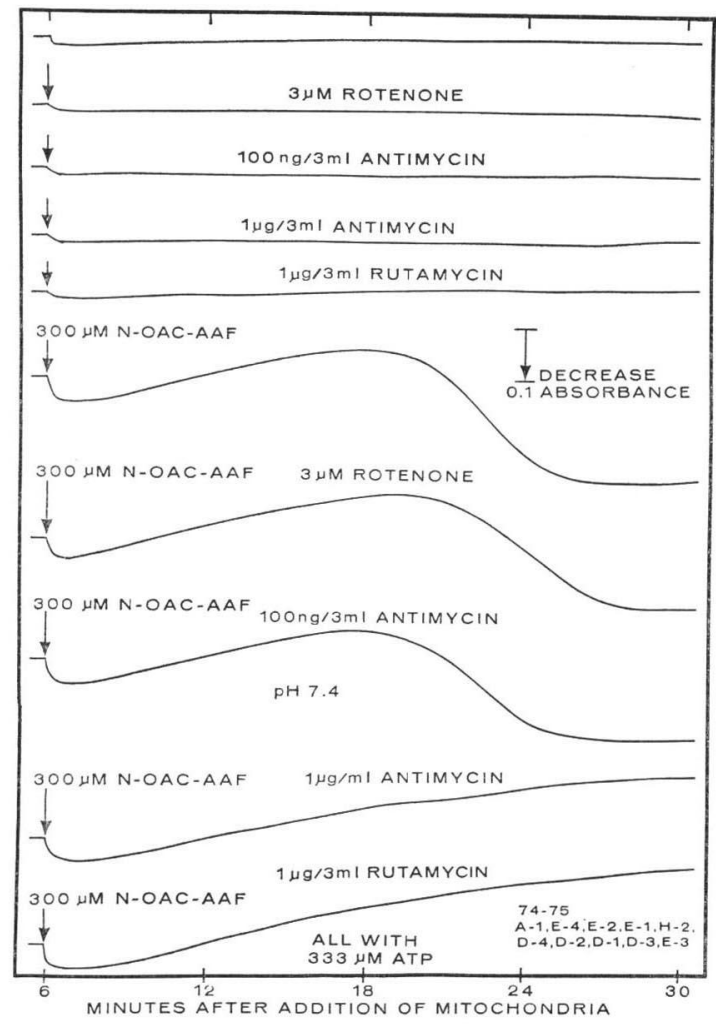

It is also seen in Fig. 3 that the ATP energized effect was inhibited by rutamycin.

The classical uncoupler of oxidative phosphorylation 2,4-dinitrophenol (DNP), also inhibited the ATP energized volume change (data not shown).

The addition of the thiol reagent showdomycin (Fig. 4) enhanced and hastened the onset of the ATP energized effect dependent on N-OAc-AAF. Acetate plus showdomycin plus ATP did not induce an ATP energized mitochondrial change. In contrast $\mathrm{N}-\mathrm{OH}-\mathrm{AAF}$ the other possible cleavage product of N-OAcAAF when combined with showdomycin produced an ATP energized mitochondrial volume change as previously reported ${ }^{2 !}$.

In Fig. 5, N-OAc-AAF was added to the mitochondrial suspension before ATP was added. The induction period required for the onset of swelling was not influenced by varying the
Fig. 4. The effect of showdomycin.

See legend of Fig. 1 for conditions. Showdomycin, where indicated, was present in the cuvette before the mitochondria were added.

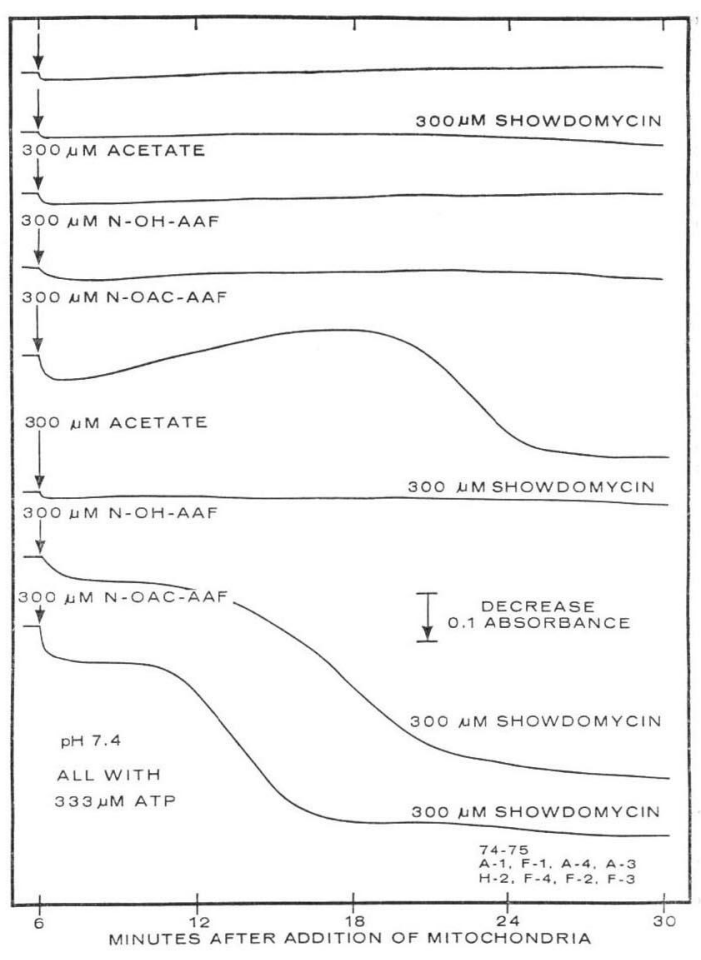

Fig. 5. Variation in the time of ATP addition. The conditions were those described by the legend of Fig. 1 except as indicated below. The addition of N-OAc-AAF is indicated by the first arrow of each graph. The addition of ATP (tris salt) in a volume of $0.05 \mathrm{ml}$ by means of the adding-mixing device is indicated by the second arrow.

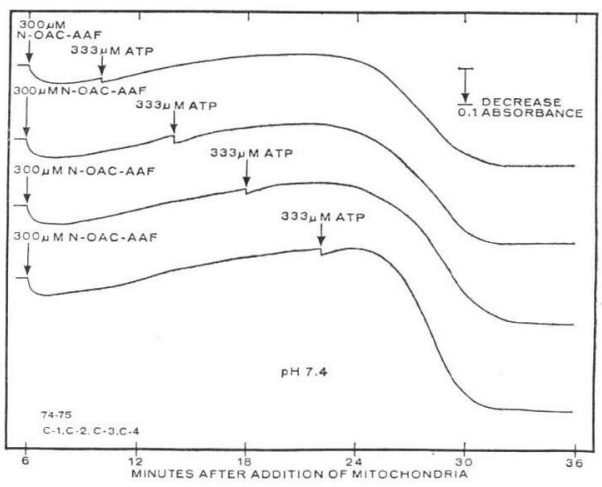


interval of time between the addition of N-OAc-AAF and the addition of ATP. The later the addition of ATP the larger the mitochondrial change.

N-OAc-AAF induced less initial ATPase activity than N-OH-AAF (Table 1). Showdomycin stimulated the ATPase activity and ATP energized volume change induced by N-OAc-AAF (Fig. 4 and Table 1). Although showdomycin was a requirement for the ATP energized volume change induced by N-OH-AAF (Fig. 4) showdomycin did not appreciably alter the ATPase activity induced by N-OH-AAF (Table 1).

Sulfate ion stimulated the ATP energized mitochondrial volume change induced by $\mathrm{N}$ OAc-AAF (Fig. 2) but did not alter the ATPase activity induced by N-OAc-AAF (Table 1).

State 3 mitochondrial respiration and DNP uncoupled respiration were inhibited by $100 \mu \mathrm{M}$

Fig. 6. Effect of N-OAc-AAF on mitochondria respiration.

The final concentrations were; sucrose $225 \mathrm{~mm}$, tris chloride $\mathrm{pH} 7.420 \mathrm{~mm}$, tris phosphate $\mathrm{pH}$ $7.410 \mathrm{~mm}$, tris $\beta$-hydroxybutyric acid $\mathrm{pH} 7.4$ $12 \mathrm{~mm}$, potassium chloride $20 \mathrm{~mm}$, magnesium chloride $5 \mathrm{~mm}$. Either ADP, DNP or N-OAcAAF (in acetone) was added in a volume of $0.02 \mathrm{ml}$ via a micro syringe fitted with a stainless steel needle ( 20 gauge) as indicated by arrows. The final volume of the system was $3 \mathrm{ml}$. The mitochondrial protein concentration was 1.0 $\mathrm{mg} / \mathrm{ml}$.

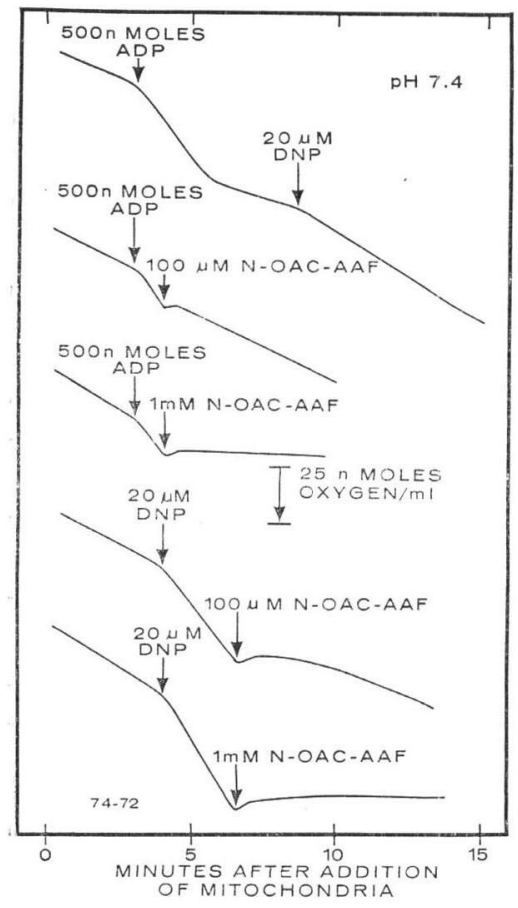

Table 1. ATPase activity of N-OAc-AAF (in $\mathrm{nmoles} \mathrm{Pi} / \mathrm{ml} / \mathrm{min}$ )

\begin{tabular}{c|c|c|c}
\hline Additions & $\begin{array}{c}\text { ATP } \\
(333 \mu \mathrm{M})\end{array}$ & $\begin{array}{c}\text { N-OAc-AAF } \\
(300 \mu \mathrm{M})\end{array}$ & $\begin{array}{c}\text { N-OH-AAF } \\
(300 \mu \mathrm{M})\end{array}$ \\
\hline 23 & 50 & 84 \\
$\begin{array}{c}\text { Showdomycin } \\
(300 \mu \mathrm{M})\end{array}$ & 24 & 90 & 82 \\
\begin{tabular}{c} 
Sulfate $(2 \mathrm{mM})$ \\
\hline
\end{tabular} & 22 & 50 & \\
\hline
\end{tabular}

The assay procedure for phosphate employing the specially designed cuvettes is described in "Materials and Methods." The final volume of the system was $15 \mathrm{ml}$. The initial velocity of ATP hydrolysis was taken from the first linear portion of the curve.

Fig. 7. Thin-layer chromatographic analysis of N-OAc-AAF incubated with mitochondria.

See "Materials and methods" for conditions of incubation and extraction.

The spots from left to right are as follows:

1. Boiled mitochondria, 0.5-min incubation.

2. Boiled mitochondria, 30-min incubation.

3. Native mitochondria, $0.5-\mathrm{min}$ incubation.

4. Native mitochondria, 30-min incubation.

The prominent spots were those associated with N-OAc-AAF (Rf 0.90 ) and N-OH-AAF (Rf 0.46 with tailing). In order to quantify the prominent spots, the absorbant ( 1 $\mathrm{cm}$ ) was scraped off, the developed plate, about each spot. The organic materials were eluted from the absorbant with $2 \mathrm{ml}$ of methanol (distilled). After centrifugation the absorbance at $275 \mathrm{~nm}$ was read in quartz cuvettes with a $1-\mathrm{cm}$ light path. $275 \mathrm{~nm}$ corresponds to or is close to an absorption maxima of the two compounds. The absorbance of spots of N-OAc-AAF from lefit to right are: 508, 481, 437,81 . The absorbance of spots of N-OH-AAF from left to right are: $11,13,8,141$. The $\mathrm{N}-\mathrm{OH}-$ AAF always had characteristic trailing in the solvent system used. The other spots, which may be metabolic or artifactual, remain unidentified, and were not present in the N-OAc-AAF. 
N-OAc-AAF and respiration was completely blocked by $1 \mathrm{~mm}$ N-OAc-AAF when the mitochondrial concentration was $1.25 \mathrm{mg}$ mitochondrial protein per $\mathrm{ml}$ (Fig. 6). Inhibition was immediate and sharp. The data does not reveal which coupling sites were blocked.

Rat liver mitochondria mediated an enzymic cleavage of N-OAc-AAF to yield N-OH-AAF (Fig. 7).

In all instances (Figs. 1 and 3) when N-OAc-AAF was present either alone or in combination with other reagents (e.g. DNP or antimycin) so that no ATP energized increase in mitochondrial volume was observed there was a decided decrease in volume as indicated by an increase of absorbance at $520 \mathrm{~nm}$.

\section{Discussion}

Neither ATP alone nor N-OAc-AAF alone was capable of inducing an increase in mitochondrial volume, however, such an effect was observed when these two agents were used in combination (Fig. 1). This observation held true whether the N-OAc-AAF was prepared as described by GUTMANN and ERICKSON ${ }^{27)}$ or by the method of LOTLIKAR et al. ${ }^{14)}$ or by the method described in this report. Since the ATP energized effect was inhibited by rutamycin (Figs. 1 and 3) and such inhibition is characteristic of ATP energized mitochondrial processes involving the machinery of oxidative phosphorylation ${ }^{29,30,31)}$ it follows that N-OAc-AAF interacted with the machinery of oxidative phosphorylation.

The induction period of the ATP energized phenomenon (Fig. 5) did not vary when the time between the addition of mitochondria and the subsequent addition of ATP was progressively increased. This suggests that the induction period prior to the observable decrease in absorbancy was due to a reaction between mitochondria and N-OAc-AAF. Once this reaction had taken place to an adequate extent, ATP was able to energize the volume change. Furthermore, ATP is not required for the reaction to take place during the induction period. As N-OAc-AAF set up an ATPase activity in the mitochondria (Table 1) the amount of ATP present at the end of the induction period, presumably was greater the greater the delay between the addition of mitochondria and the addition of ATP. This would explain the graded increase in response seen in Fig. 5. This also explains why reagents (e.g. DNP, the high level of antimycin) which set up substantial ATPase activity prevent the ATP energized volume change phenomenon induced by N-OAc-AAF.

Whenever N-OAc-AAF was added to the mitochondria and no ATP energized mitochondrial expansion took place (i.e. N-OAc-AAF alone, N-OAc-AAF plus ATP plus one of rutamycin, a high concentration of antimycin, or DNP) a definite delayed mitochondrial contraction was observed. A slight contraction was also characteristic of the induction period previously discussed. These observations are all compatible with a reaction between N-OAc-AAF and mitochondria occurring during the induction period.

Showdomycin stimulated both the ATP energized mitochondrial volume change (Fig. 4) and ATPase activity (Table 1) induced by N-OAc-AAF. In contrast while showdomycin was a requirement for the ATP energized mitochondrial volume change induced by N-OH-AAF (Figs. 1 and 4), showdomycin had little effect on the ATPase activity induced by N-OH-AAF (Table 1). Acetate alone (Fig. 1) or in combination with showdomycin (Fig. 4) did not induce an ATP energized mitochondrial volume change. Thus the reaction of N-OAc-AAF during the induction period could not be attributed to two of its possible products viz. N-OH-AAF and acetate. Nevertheless the enzymic conversion of N-OAc-AAF to N-OH-AAF was demonstrated (Fig. 7). Thus in addition to N-OAc-AAF undergoing a cleavage reaction which yielded NOH-AAF there is some other reaction involving N-OAc-AAF and the machinery of oxidative phosphorylation.

Like N-OH-AAF ${ }^{2}$ N-OAc-AAF was a respiratory inhibitor (Fig. 6). The sharp response reflected the easy penetration of the mitochondria by a non-polar, reactive substance. 
Sulfate ion increased the rate of the ATP independent reaction between mitochondria and the electrophile N-OAc-AAF which took place during the induction period as the induction period was decrease but the ATPase activity remained unaltered (Fig. 2, Table 1). It is cogent that sulfate ion has been implicated as a requirement for liver carcinogenesis induced by $\mathrm{N}$ $\mathrm{OH}-\mathrm{AAF}^{10,11,12,13)}$.

The sulfotransferase which converts $\mathrm{N}-\mathrm{OH}-\mathrm{AAF}$ to $\mathrm{N}-\mathrm{OSO}_{2} \mathrm{OH}-\mathrm{AAF}$ has been located in the cytosol of the rat liver ${ }^{11,32,13,33)}$. When $\mathrm{N}-\mathrm{OSO}_{2} \mathrm{OH}-\mathrm{AAF}$ was generated in vitro it did not bind to nuclear nucleic acid enclosed by the nuclear membrane ${ }^{13)}$. If binding to nucleic acid is considered essential for carcinogenesis the route whereby $\mathrm{N}-\mathrm{OSO}_{2} \mathrm{OH}-\mathrm{AAF}$ affects the nuclear genome is moot. One should recognize, however, the data of Weisburger et al. ${ }^{13)}$ does not eliminate all in vivo possibilities. On the other hand, if mitochondrial disturbance is significant for carcinogenesis the in vitro data with N-OAc-AAF presented in this report supports such a thesis and directs attention to our unitary theory for carcinogenesis ${ }^{2,6)}$.

While both AAF and N-OH-AAF produced tumors in rat ZYMBAL's and mammary glands as well as liver, N-OH-AAF sulfotransferase was found in only in liver ${ }^{11,32,331}$. Although rat embryo fibroblasts were transformed by N-OH-AAF the sulfotransferase was not detected in these cells ${ }^{34}$. The macromolecular binding theory accordingly requires the replacement of $\mathrm{N}$ $\mathrm{OSO}_{2} \mathrm{OH}-\mathrm{AAF}$ by another electrophilic agent ${ }^{7)}$ in certain instances. On the other hand, because $\mathrm{N}-\mathrm{OH}-\mathrm{AAF}$ as well as N-OAc-AAF interacted with the machinery of oxidative phosphorylation it is our alternative suggestion, that $\mathrm{N}-\mathrm{OH}-\mathrm{AAF}$ itself may be the ultimate carcinogen in rat ZYMBAL's and mammary glands and embryo fibroblasts. YOST, GUTMANN and RYDELL ${ }^{35)}$ found that while both $\mathrm{N}$-hydroxy-N-acetyl-3-aminofluorene and $\mathrm{N}$-acetoxy- $\mathrm{N}$-acetyl-3-aminofluorene were carcinogenic in the rat the $\mathrm{N}$-acetoxy compound did not behave like a model ultimate electrophilic carcinogenic agent and generate the arylamidonium moiety. This is compatible with our suggestion that the ultimate carcinogen may in certain circumstances be the hydroxamic acid.

The search for an alternative electrophile to $\mathrm{N}-\mathrm{OSO}_{2} \mathrm{OH}-\mathrm{AAF}$ has recently been extended to include $\mathrm{N}$-acetoxy-2-aminofluorene (N-OAc-AF) ${ }^{36,37,38 !}$. N-OAc-AF is believed to be a potent electrophile derived enzymically from N-OH-AAF via a transacylase reaction. Our data with N-OAc-AAF certainly raises the possibility that N-OAc-AF could disturb the machinery of oxidative phosphorylation. No direct experimentation with N-OAc-AF is yet possible as the successful synthesis of N-OAc-AF remains to be accomplished ${ }^{36,381}$.

The assignment of the role of model ultimate carcinogen to N-OAc-AAF rather than to $\mathrm{N}-\mathrm{OH}-\mathrm{AAF}$ depends strongly upon the greater local carcinogenic potency of N-OAc-AAF (subcutaneous injection in the rat) relative to $\mathrm{N}-\mathrm{OH}-\mathrm{AAF}^{23,7)}$. This conclusion is not unequivocal because the reported greater potency of N-OAc-AAF could be due to the lesser polarity of $\mathrm{N}-\mathrm{OAc}-\mathrm{AAF}$ (an anhydride) relative to $\mathrm{N}-\mathrm{OH}-\mathrm{AAF}$ (a hydroxamic acid). Thus N-OAc-AAF could penetrate the cell more readily. Our observed $\mathrm{Rf}$ values for N-OAc-AAF, AAF, and $\mathrm{N}-\mathrm{OH}-\mathrm{AAF}, 0.92,0.60$ and 0.46 respectively, are in agreement with these relative polarities. The assignment to $\mathrm{N}-\mathrm{OH}-\mathrm{AAF}$, the role of metabolite involved in carcinogenesis is also based upon the greater local carcinogenic potency of N-OH-AAF (by subcutaneous injection in the rat, mouse, hamster, rabbit and guinea pig) relative to $\mathrm{AAF}^{23,71}$. This conclusion is strengthened by the type of argument used above, because even though N-OH-AAF (a hydroxamic acid) is more polar and, therefore, presumably less permeant through cellular membranes than AAF (an acetamide), N-OH-AAF has a greater carcinogenic potential. In this instance, a chance correlation between the ability of an agent to penetrate the cell and carcinogenicity does not exist.

\section{References}

1) Hadler, H. I. \& A. C. Kryger: K-Region fission and addition products of 7,12-dimethylbenz(a) anthracene. J. Org. Chem. 25: 1896 1901, 1960 
2) Hadler, H. I.; B. G. Daniel \& R. D. Pratt: The induction of ATP energized mitochondrial volume changes by carcinogenic N-hydroxy-N-acetyl-aminofluorenes when combined with showdomycin. A unitary hypothesis for carcinogenesis. J. Antibiotics 24: 405 417, 1971

3) Hadler, H. I.; B. G. Daniel, J. Demetriou \& R. C. Pratt: The induction of ATP energized mitochondrial volume changes by showdomycin when combined with $4^{\prime}, 8^{\prime}$-dihydroxy-1,2,5,6-dibenz9,10-anthraquinone, a metabolite of the carcinogenic polynuclear hydrocarbon dibenz(a, h)anthracene. J. Antibiotics 24: 835 845, 1971

4) HAdLeR, H. I. \& B. G. DANIEL: The in vitro interaction of a metabolite of N-acetyl-4-aminobiphenyl with rat liver mitochondria. Cancer Res. 32: 1037 1041, 1972

5) Hadler, H. I. \& B. G. Daniel: A correlation between the carcinogenicity of isomeric N-hydroxy$\mathrm{N}$-acetylaminofluorenes and their in vitro effect on mitochondria. Cancer Res. 33: 117 122, 1973

6) HAdler, H. I.: Carcinogenesis, oxidative phosphorylation and mitochondria. Medikon 3: 22 26, 1974

7) Miller, J.A.: Carcinogenesis by chemicals (An Overview-G. H. A. Clowes Memorial Lecture). Cancer Res. 30: 559 576, 1970

8) Miller, J.A. \& E. C. Miller: Guest editorial. Chemical carcinogenesis: mechanisms and approaches to its control. J. Natl. Cancer Inst. 47: V xiv, 1971

9) DeBaun, J. R.; Y. J. Rowley, E. C. Miller \& J. A. Miller: Sulfotransferase activation of Nhydroxy-2-acetylaminofluorene in rodent livers susceptible and resistant to this carcinogen. Proc. Soc. Expt1. Biol. Med. 129: 268 273, 1968

10) DeBaun, J. R.; J. Y. R. Smith, E. C. Miller \& J. A. Miller: Reactivity in vivo of the carcinogen N-hydroxy-2-acetylaminofluorene: Increase by sulfate ion. Science 167: 184 186, 1970

11) DeBaun, J. R.; E. C. Miller \& J. A. Miller: N-Hydroxy-2-acetylaminofluorene sulfotransferase: Its probable role in carcinogenesis and in protein-(methion-S-yl) binding in rat liver. Cancer Res. 30: $577 \sim 595,1970$

12) Gutmann, H. R.; D. Malejka-Giganti, E. J. Barry \& R. E. Rydell: On the correlation between hepatocarcinogenicity of the carcinogen, $\mathrm{N}$-2-fluorenylacetamide, and its metabolic activation by the rat. Cancer Res. 32: 1554 1561, 1972

13) Weisburger, J. H.; R. S. Yamamoto, G. M. Williams, P. H. Grantham, T. Matsushima \& E. K. Weisburger: On the sulfate ester of N-hydroxy-N-2-fluorenylacetamide as a key ultimate hepatocarcinogen in the rat. Cancer Res. 32: 491 500, 1972

14) Lotlikar, P. D.; J. D. Scribner, J. A. Miller \& E. C. Miller: Reactions of esters of aromatic N-hydroxy amines and amides with methionine in vitro: A model for in vivo binding of amine carcinogens to protein. Life Sci. 5: 1263 1269, 1966

15) Maher, V. M.; E. C. Miller, J. A. Miller \& W. Szybalski: Mutations and decrease in density of transforming DNA produced by derivatives of the carcinogens 2-acetylaminofluorenes and $\mathrm{N}$ methyl-2-aminoazobenzene. Mol. Pharmacol. 4: 411 426, 1968

16) Scribner, J. D.; J. A. Miller \& E.C. Miller: Nucleophilic substitution on carcinogenic Nacetoxy-N-arylacetamides. Cancer Res. 30: 1570 1579, 1970

17) Kriek, E.; J. A. Miller, V. Juhl \& E. C. Miller: 8-(N-2-Fluorenylacetamido)guanosine and arylamidation reaction product of guanosine and the carcinogen $\mathrm{N}$-acetoxy-N-2-fluorenylacetamide in neutral solution. Biochemistry 6: 177 182, 1967

18) Grunberger, D. \& I. B. Weinstein: Modification of ribonucleic acid by chemical carcinogens. III. Template activity of polynucleotides modified by $\mathrm{N}$-acetoxy-2-acetylaminofluorene. J. Biol. Chem. 246: 1123 1128, 1971

19) Levine, A. F.; L. M. Fink, I. B. Weinstein \& D. Grunberger: Effect of N-2-acetylaminofluorene modification on the conformation of nucleic acids. Cancer Res. 34: 319 327, 1974

20) Fuchs, R.P.P. \& M.P. Daune: Dynamic structure of DNA modified with the carcinogen Nacetoxy-N-2-acetylaminofluorene. Biochemistry 13: 4435 4440, 1974

21) Lotlikar, P. D. \& L. Luha: Acetylation of the carcinogen N-hydroxy-2-acetylaminofluorene by acetyl coenzyme A to form a reactive ester. Mol. Pharmacol. 7: 381 388, 1971

22) Bartsch, H.; J. A. Miller \& E. C. Miller: One-electron non-enzymatic and enzymatic oxidation products of various carcinogenic aromatic acethydroxamic acids. Biochim, Biophys. Acta 273: $40 \sim 51,1972$

23) Miller, J.A. \& E. C. Miller: The metabolic activation of carcinogenic aromatic amines and amides. Progr. Exptl. Tumor Res. 11: 273 301, 1969

24) Falcone, A. B. \& H. I. Hadler: Action of gramicidin on mitochondria. I. Ion-dependent 
mitochondrial volume changes energized by adenosine 5'-triphosphate. Arch. Biochem. Biophys. 124: $91 \sim 109,1968$

25) Hadler, H. I. \& A. B. Falcone: Action of gramicidin on mitochondria. II. Ion-dependent mitochondrial volume changes energized by substrate oxidation. Arch. Biochem. Biophys. 124: $110 \sim 114,1968$

26) Hadler, H. I.; B. E. Claybourn \& T. P. Tschang: The mode of action of dinitrophenol revealed by mitochondrial volume changes requiring rotenone or antimycin or dinitrophenol with showdomycin. J. Antibiotics 21: 575 581, 1968

27) Gutmann, H. R. \& R. R. Erickson: The conversion of the carcinogen N-hydroxy-2-fluorenylacetamide to $o$-amidophenols by rat liver in vitro: An inducible enzymatic reaction. J. Biol. Chem. 244: $1729 \sim 1740,1969$

28) Slater, E. C.: In Methods in Enzymology 10: 50, 1967

29) Lardy, H. A.; D. Johnson \& W. C. MCMurray: Antibiotics as tools for metabolic studies. I. A survey of toxic antibiotics in respiratory, phosphorylative and glycolytic systems. Arch Biochem. Biophys. 78: 587 597, 1958

30) Lardy, H. A.; P. Witonsky \& D. Johnson: Antibiotics as tools for metabolic studies. IV. Comparative effectiveness of oligomycins A, B, C, and rutamycin as inhibitors of phosphoryl transfer reactions in mitochondria. Biochemistry 4: 552 554, 1965

31) Henderson, P. J. \& H. A. Lardy: Antibiotic inhibition of mitochondrial energy-transfer reactions. Antimicr. Agents \& Chemoth.-1969: 18 27, 1970

32) IRVing, C. C.; D. G. JANS \& L. T. Russel: Lack of N-hydroxy-2-acetylaminofluorene sulfotransferase activity in the mammary gland and ZyMBAL's gland of the rat. Cancer Res. 31: 387 391, 1971

33) Malejka-Giganti, D.; H. R. Gutmann \& R. E. Rydell: Mammary carcinogenesis in the rat by topical application of fluorenylhydroxamic acids. Cancer Res. 33: 2489 2497, 1973

34) Sekely, L. I.; D. Malejka-Giganti, H. R. Gutmann \& R. E. Rydell: Malignant transformation of rat embryo fibroblasts by carcinogenic fluorenylhydroxamic acids in vitro. J. Natl. Cancer Inst. 50: $1337 \sim 1345: 1973$

35) Yost, Y.; H. R. Gutmann \& R. E. Rydell: The carcinogenicity of fluorenylhydroxamic acids and $\mathrm{N}$-acetoxy-N-fluorenylacetamides for the rat as related to the reactivity of the esters toward nucleophiles. Cancer Res. 35: 447 459, 1975

36) Bartsch, H.; M. Dworkin, J. A. Miller \& E. C. Miller: Electrophilic N-acetoxyaminoarenes derived from carcinogenic $\mathrm{N}$-hydroxy-N-acetylaminoarenes by enzymatic deacetylation and transacetylation in liver. Biochim. Biophys. Acta 286: 272 298, 1972

37) Bartsch, H.; C. Dworkin, E. C. Miller \& J. A. Miller: Formation of electrophilic Nacetoxyarylamines in cytosols from rat mammary gland and other tissues by transacetylation from the carcinogen N-hydroxy-4-acetylaminobiphenyl. Biochim. Biophys. Acta 304: 42 55, 1973

38) KING, C. M.: Mechanism of reaction, tissue distribution, and inhibition of arylhydroxamic acid acyltransferase. Cancer Res. 34: 1503 1515, 1974 